\title{
Decreasing Levels of the cdk5 Activators, p25 and p35, Reduces Excitotoxicity in Striatal Neurons
}

\author{
Kevin H.J. Park ${ }^{\mathrm{a}}$, Ge Lu ${ }^{\mathrm{a}}$, Jing Fan ${ }^{\mathrm{b}}$, Lynn A. Raymond ${ }^{\mathrm{b}, \mathrm{c}}$ and Blair R. Leavitt ${ }^{\mathrm{a}, \mathrm{c}, *}$ \\ ${ }^{a}$ Centre for Molecular Medicine and Therapeutics, Department of Medical Genetics, Child and Family Research \\ Institute, University of British Columbia, Vancouver, BC, Canada \\ ${ }^{\mathrm{b}}$ Department of Psychiatry, Brain Research Centre, University of British Columbia, Vancouver, BC, Canada \\ ${ }^{\mathrm{c}}$ Department of Medicine, Division of Neurology, University of British Columbia, Vancouver, BC, Canada
}

\begin{abstract}
Huntington's disease (HD) is a progressive neurodegenerative disorder caused by an expanded CAG trinucleotide repeat sequence in the huntingtin gene. The resulting poly-glutamine expansion in the huntingtin protein imparts a novel toxic gain of function causing selective loss of medium spiny neurons (MSNs) in the striatum. Although the exact mechanism of cell death is unclear, recent evidence suggests involvement of NMDA-receptor mediated excitotoxicity and aberrant cyclin dependent kinase 5 (cdk5) activity in striatal cells undergoing neurodegeneration. In this study we directly tested the effect of reduced levels of $\mathrm{p} 25$ and $\mathrm{p} 35$, two proteins required for cdk5 activation, on striatal neurodegeneration using mice with targeted deletion of p35. Quinolinic acid (QA) injected into the striatum of mice causes NMDA-receptor mediated cell death, and these QAinduced striatal lesions were examined in $\mathrm{p} 35$ hemizygous null (p35+/-) and wildtype (WT) mice. Striatal QA lesion volumes were $30 \%$ smaller in p35+/- mice than in WT mice. Furthermore, primary neuronal cultures of MSNs from P0 p35+/- pups displayed 33\% less apoptotic neurons following NMDA treatment than those from WT pups. Examination of YAC128 mouse model of HD showed elevated p25 levels in striatum following intrastriatal QA injection. Our findings provide direct evidence for $\mathrm{p} 25$ and p35 involvement in excitotoxic neurodegeneration of MSNs and suggest a role for the cdk5 pathway in HD striatal neurodegeneration.
\end{abstract}

Keywords: Huntington's disease, medium spiny neuron, striatum, YAC128, cdk5, p25, quinolinic acid, NMDA, excitotoxic, neurodegeneration

\section{ABBREVIATIONS}

HD Huntington's disease

MSN medium spiny neuron

cdk5 cyclin-dependent kinase 5

QA quinolinic acid

YAC128 YAC128 mouse model of HD

PM plating medium

mHtt mutant huntingtin

WT wildtype

\footnotetext{
${ }^{*}$ Correspondence to: Blair R. Leavitt, MDCM, FRCPC (Neurology), Centre for Molecular Medicine and Therapeutics, Department of Medical Genetics, Child and Family Research Institute, University of British Columbia, Vancouver, V5Z4H4 BC, Canada. Tel.: +1 604 875 3801; Fax: +1 604875 3840; E-mail: bleavitt@cmmt.ubc.ca.
}

\section{INTRODUCTION}

Huntington's disease (HD) is a neurodegenerative genetic disorder caused by polyglutamine expansion in the $\mathrm{N}$-terminal domain of the huntingtin protein [1]. A major pathological hallmark of the disease is selective striatal degeneration resulting from the loss of medium spiny neurons in the caudate/putamen [2]. Although the pathological mechanism of the selective neuronal loss is unclear, studies from full-length mutant huntingtin ( $\mathrm{mHtt}$ ) transgenic mice have demonstrated increased sensitivity to NMDA excitotoxicity in the striatum [3]. NMDA-receptor activity, mitochondrial function, and intracellular IP3 receptors are altered in the presence of $\mathrm{mHtt}$ and lead to increased 
intracellular calcium load [4]. Calcium influx leads to activation of calcium-mediated proteases including various calpains [5], which are increased in HD brains and YAC128 mice [6, 7].

Cyclin-dependent kinase $5(\operatorname{cdk} 5)$ is a prolinedirected serine/threonin kinase that is activated when bound by its neuronally enriched activators p35 or p39 [8-11]. Cdk5 plays an important role in neuronal migration, neuronal differentiation, axon guidance, axonal transport, and synaptic plasticity [12-14]. On the other hand, cdk5 dysregulation resulting from cdk5 activation by $\mathrm{p} 25$ has been shown to be involved in neurodegeneration associated with a number of neurological disorders including Alzheimer's and Parkinson's diseases [13-16]. Under excitotoxic conditions, p35 is cleaved by calpain resulting in the formation of p25 [17, 18]. p25 lacks the n-terminal myristoylation signal, resulting in altered localization and abnormal protein interaction which may lead to neurodegeneration $[16,18,19]$.

Recently, a number of studies have highlighted increased p25 in HD models of striatal neurodegeneration. In an immortalized striatal cell line, presence of mHtt increases D1-mediated cell death which was accompanied by an increase in p25 level [20]. In a rat model study, 3-NP mediated striatal lesion was accompanied by an increase in p25 [21]. In this study we examined the impact of reduced p25 and p35 levels on excitotoxicity-mediated striatal neurodegeneration using p35 hemizygous null (p35+/-) mice. With a $50 \%$ reduction in $\mathrm{p} 35$ level, these mice show decreased p25 levels [22]. We report that p35+/- mice show $30 \%$ smaller striatal quinolinic acid (QA) lesions and medium spiny neurons (MSNs) from these mice show a 33\% reduction in NMDA-mediated apoptosis in culture. Furthermore, we report that striatum from the YAC128 mouse model of HD have an increased p25 response following intrastriatal QA injection. Our findings suggest that reduction of cdk5 activators, p25 and p35, maybe neuroprotective in HD striatal neurodgeneration. Further experiments are necessary to determine the role of p25 in HD neurodegeneration.

\section{MATERIALS AND METHODS}

\section{Animals}

YAC128 (FVB background; [26]) and p35+/- (B6 background; [22]) mice were used in this study. Mice were maintained on a 12-hour light/dark cycle with free access to food and water. All animal protocols were approved by the Animal Care Committee at the University of British Columbia.

\section{Intrastriatal QA injections}

All surgical procedures were performed in accordance with University of British Columbia Animal Care Committee guidelines. QA (Sigma) was dissolved into 0.1 M PBS. Mice were anesthetized with $1.5 \%$ isoflurane and received intrastriatal injections of QA. Coordinates of the injection site are as follows: $0.8 \mathrm{~mm}$ posterior to Bregma, $1.8 \mathrm{~mm}$ lateral from the midline and $3.5 \mathrm{~mm}$ below the dorsal surface of the neocortex. For YAC128 vs. WT study (FVB mice), 8 nmoles of QA in $0.4 \mu \mathrm{l}$ volume was injected. And for p35+/- vs. WT study (B6), 10 nmoles of QA in $0.5 \mu \mathrm{l}$ volume was injected.

\section{QA lesion volume analysis}

All quantitative analysis was done blind with respect to genotype. Five days after intrastriatal QA injection, mice were terminally anesthetized by intraperitoneal injection of $2.5 \%$ avertin and perfused with $3 \%$ paraformaldehyde (PFA) in PBS. The brains were stored in $3 \%$ PFA in PBS for $24 \mathrm{~h}$ at $4{ }^{\circ} \mathrm{C}$ then removed and stored in PBS. Before sectioning, brains were transferred to a $30 \%$ sucrose solution containing $0.08 \%$ sodium azide in PBS, left overnight then mounted using Tissue-TEK O.C.T. compound (Sakura). Mouse brains were cut using a cryostat (MicromHM500M, Richard-Allan Scientific) and coronal sections (25 $\mu \mathrm{m})$ spaced $150 \mu \mathrm{m}$ apart were stained with Fluro-Jade (Histo-Chem). The perimeter of the striatal lesion for each section was traced using StereoInvestigator software (Microbrightfield). For assessment of the number of Fluoro-Jade-positive cells, a $25 \times 25 \mu \mathrm{m}$ counting frame was randomly placed within the delineated lesion area and then systematically moved through the striatum. The number of FluoroJade-positive cells was then determined by StereoInvestigator software (Microbrightfield).

\section{Primary neuronal cultures}

Striatal cultures were prepared from postnatal day 0-1 (P0-P1) wild-type C57BL/6 mice or p35+/mice as described previously [3] and plated at a density of approximately $2 \times 10^{5}$ cells/well in 24-well plates with Poly-D-Lysine $(250 \mu \mathrm{g} / \mathrm{ml})$ precoated $12 \mathrm{~mm}$ glass coverslips. Neuronal cultures were grown in serum-free plating medium (PM) (B27, 
penicillin/streptomycin, $\alpha$-glutamine, Gibco's Neurobasal medium), maintained in a humidified $37^{\circ} \mathrm{C}$ incubator with $5 \% \mathrm{CO}_{2}$, and refreshed every 5 days by replacing half of the medium.

\section{NMDA-induced toxicity}

Striatal neurons (DIV 8-9) cultured in 24-well plates were incubated with $100 \mu \mathrm{M}$ NMDA or $500 \mu \mathrm{M}$ NMDA for $10 \mathrm{~min}$, washed once with warm PM, and then incubated in conditioned PM for 24 hours. Then cells were washed with PBS once and fixed with $4 \%$ PFA in PBS for 30 minutes.

\section{TUNEL assay and assessment of apoptosis}

Fixed striatal cultures on coverslips were randomly coded according to different conditions before staining to ensure that the operator was blinded during the subsequent processing and analysis of immunofluorescence. Striatal cultures were stained with TUNEL (Roche) reagent and $10 \mu \mathrm{M}$ Hoechst 33342 as described previously [23]. Coverslips were then mounted on slides with Fluoromount-G. The percentage of apoptotic cell death for cultured striatal neurons was assessed by counting the numbers of TUNEL positive cells (green fluorescent channel), which also showed condensed and blebbed nuclear morphology in the blue fluorescent channel, then dividing by the total number of Hoechst positive cell nuclei (blue channel) and multiplying by 100 . At least 1000 neurons were counted per condition for each experiment (duplicate coverslips for each condition). Representative photomicrographs of immunofluorescent striatal cultures were taken using $63 \times$ lens by Northern Eclipse software or Axio Vision Rel. using a Zeiss Axiophot or Axiovert zoom microscope.

\section{Western blotting}

Western blotting was performed as reported previously [24] using supernatants from microdissected striatum. Protein quantities were determined using Qubit quantitation platform (Invitrogen). Samples were electrophoresed, transferred to nitrocellulose (Protran, Schleicher and Schuell, Dassel, Germany). Band density was analyzed using Quantity One software (Biorad, Mississauga, ON). Beta-tubulin (Sigma) was used as loading control. P35 antibody (C9) was obtained from Santa Cruz Biotechnology and spectrin antibody was obtained from Millipore.
HRP-conjugated secondary antibodies were purchased from Southern Biotech.

\section{Statistics}

Two-way ANOVA, One-way ANOVA, Bonferroni post-test, and two-tailed unpaired $t$-test were performed where appropriate using Prism 4 for Macintosh (Prism v. 4.0, GraphPad Software Inc., San Diego, CA).

\section{RESULTS}

Striatal neurons generated from p35+/- mice have decreased sensitivity to NMDA-mediated excitotoxicity

To determine whether MSN sensitivity to NMDAreceptor mediated excitotoxicity is altered in p35+/mice, we generated primary MSN cultures from postnatal day 0 pups of $\mathrm{p} 35+/-$ and WT mice. These primary neuron cultures were challenged with NMDA and apoptotic neurons were measured by counting TUNEL positive neurons (Fig. 1A). p35+/- MSN showed $33 \%$ decrease in the number of cells that were positive for TUNEL compared to WT MSN when challenged with either $100 \mu \mathrm{M}$ or $500 \mu \mathrm{M}$ NMDA (Fig. 1B, Two-way ANOVA, $\mathrm{F}=25.85, p<0.0001)$. Multiple comparison post-test showed statistical difference for $500 \mu \mathrm{M}$ NMDA treatment $(p<0.05)$. Therefore, MSN from $\mathrm{p} 35+/-$ mice show reduced sensitivity to NMDAmediated excitotoxicity.

\section{Decreased sensitivity to striatal excitotoxicity in p35+/- mice}

A number of studies have reported upregulation of p25 in striatum either in the presence of mHtt or an excitotoxic chemical lesion [20, 21]. We examined the role of p25 level in excitotoxic striatal neurodegeneration using $\mathrm{p} 35+/-$ mice. These mice show approximately $50 \%$ reduction in p35 levels [22], resulting in decreased p 25 response. Using these mice, we examined the effect of lower p25 and p35 levels on striatal lesion volume following intrastriatal QA injection, an excitotoxic chemical lesion model of acute MSN death that mimics some features of the neuronal loss in HD [25].

Striatal p25 levels are increased following intrastriatal injection of QA (Suppl. Figure 1, two-way ANOVA, QA effect, $p<0.0001$; http://cmmt.ubc.ca/ kpark/park_supplementary_data.pdf). The large vari- 
A

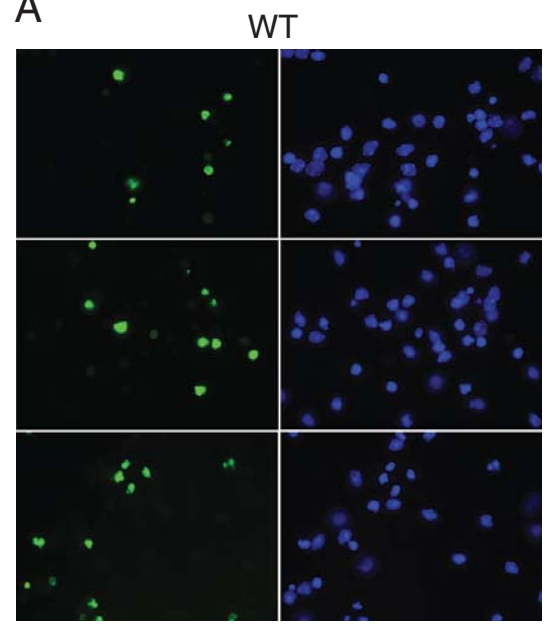

TUNEL
Hoechst
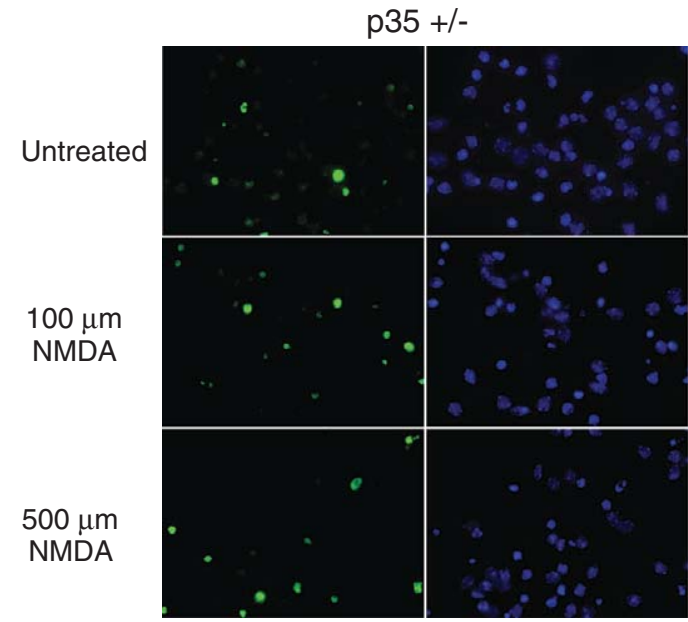

TUNEL

Hoechst

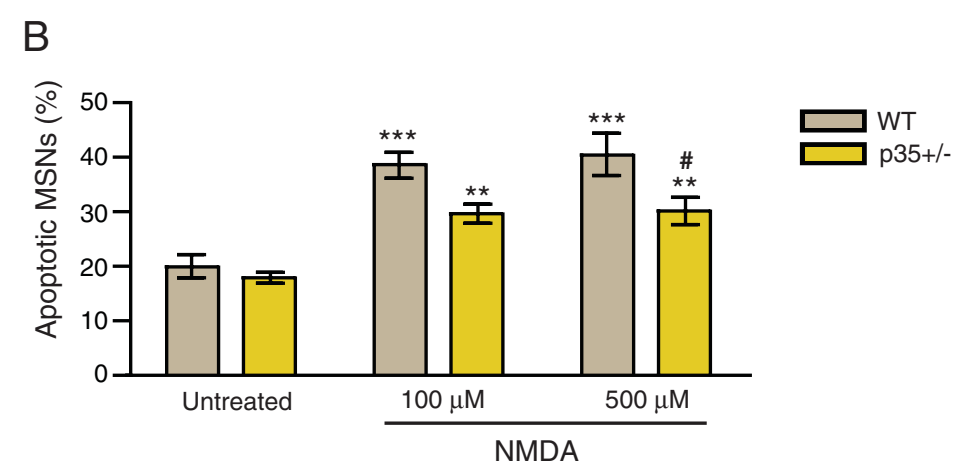

Fig. 1. Primary MSN cultures from p35+/- mice show reduced sensitivity to NMDA-mediated excitoxicity. We examined whether MSN in p35+/- mice show reduced sensitivity to NMDA-mediated excitotoxicity. (A) Primary MSN cultures were generated from P0 pups of WT and p35+/- mice were challenged with NMDA. The cultures were then TUNEL stained to identify apoptotic neurons. (B) Fewer p35+/MSN stained for TUNEL following NMDA exposure compared to the WT MSN suggesting that MSNs from p35+/- mice are less sensitive to NMDA-mediated excitotoxicity than WT MSNs (Two-way ANOVA, $p=0.0023$ for treatment, $p<0.0001$ for genotype. By Bonferroni's post-test, ${ }^{* *} p<0.01,{ }^{* *} p<0.001$ comparing NMDA treatment groups to its untreated baseline; ${ }^{*} p<0.05$, comparing p35+/- to WT with the same treatment. $n=6$ for WT, $n=5$ for $\mathrm{p} 35+/-$ ).

ability between the QA samples likely reflects QA injection quality between the samples since the variance is much lower in PBS samples. Furthermore the p25 response following QA injection is reduced in p35+/- mice. As predicted, p25 level in p35+/- mice is approximately $50 \%$ of that in wildtype mice following intrastriatal QA injection (Suppl. Figure 2; http:// cmmt.ubc.ca/kpark/park_supplementary_data.pdf).

To determine whether striatal sensitivity to excitotoxic insult is reduced in p35+/- mice, 4-month old WT or $\mathrm{p} 35+/-$ mice were injected intrastriatally with QA. The mice were sacrificed 5 days post-injection and striatal lesion volume was measured by measuring fuorojade-positive areas (Fig. 2A). p35+/- mice showed significantly smaller striatal lesion volumes compared to WT mice (Fig. 2A). Striatal QA lesion volume was approximately $30 \%$ smaller in the p35+/mice, suggesting that reductions in p25 and p35 levels mitigate striatal sensitivity to QA-mediated excitotoxicity (Fig. 2B, two-tailed $t$-test, $p=0.035$ ).

\section{Striatal excitotoxicity results in elevated p25 levels in the presence of full-length $\mathrm{mHtt}$}

It has been shown that calpain activity is increased in the striatum of 2-month old YAC128 mice, which are a transgenic mouse model of HD [7]. Since p25 is generated by calpain-mediated cleavage of $\mathrm{p} 35$, we examined 4-month old YAC128 mice to determine whether basal p25 level is elevated in the striatum. 
A
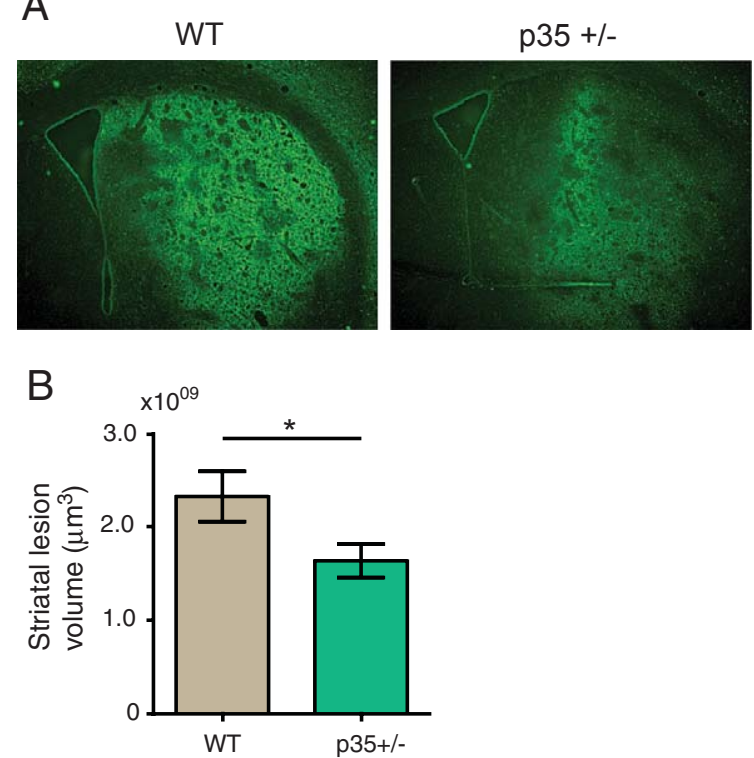

Fig. 2. Intrastriatal QA lesion volume is reduced in $\mathrm{p} 35+/-$ mice. We examined the impact of decreased p 35 and p25 on QA lesion volume using the p35+/- mice. (A) WT and p35+/- mice were intrastriatally injected with QA and the lesion volume was assessed using Fluorojade staining. (B) p35+/- mice $(n=32)$ showed approximately $30 \%$ smaller fluorojade-positive lesion volumes than WT mice $(n=35)$ (unpaired two-tailed $t$-test, $* p=0.035$ ). The data shows that decreased p25 and p35 level reduces striatal sensitivity to QAmediated excitotoxicity.
Western blot analysis of microdissected striatum did not show a basal difference in either p25 or p35 levels (Fig. 3A). The basal calpain activity was indirectly assessed by measuring the intensity of bands corresponding to the cleaved and full-length spectrin fragment on a Western blot. The ratio of cleaved and full-length spectrin band intensity was not different between YAC128 and WT mice suggesting that the calpain activity between YAC128 and wildtype mice was not different at this age in our study (Suppl. Figure 3; http://cmmt.ubc.ca/kpark/park_supplementary_ data.pdf). Although striatal pathology is minimal at 4-months of age [26], these mice display increased striatal sensitivity to QA-mediated excitotoxicity [27]. QA injection resulted in increased calpain activity compared to untreated mice as demonstrated by increase in the ratio between cleaved and full-length spectrin (Suppl. Figure 3; http://cmmt.ubc.ca/kpark/ park_supplementary_data.pdf). On the other hand, the ratios for QA injected YAC128 vs. WT were similar again suggesting that the caplain activation following QA injection is similar between YAC128 and WT mice (suppl. Figure 3; http://cmmt.ubc.ca/ kpark/park_supplementary_data.pdf). Nonetheless, Western blot analysis of QA injected YAC128 mice show increased p25 level in the striatum compared to wildtype mice (Fig. 3B, $p=0.0238$ ). The p35 level was not statistically different (Fig. 3B). Therefore at
A

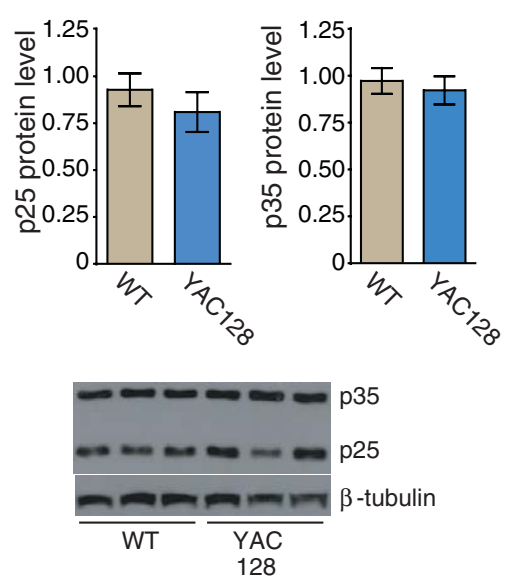

B

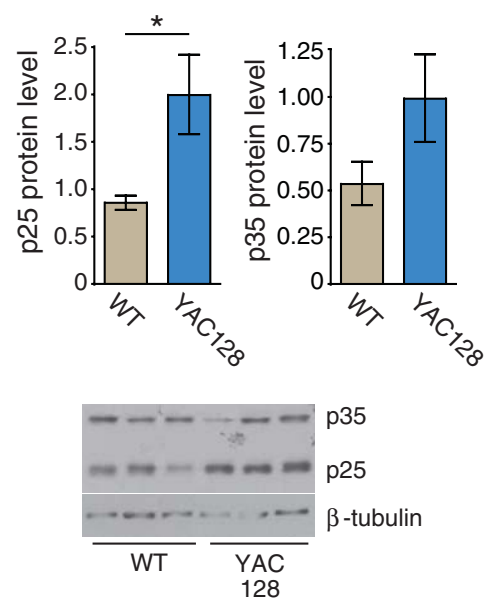

Fig. 3. p25 level in striatum is elevated in YAC128 mice in response to intrastriatal QA injection. We examined YAC128 mice to determine whether p25 level is elevated in the striatum in the presence of full-length mutant Htt. (A) Examination of 4-month old YAC128 and WT mice show that there is no difference in basal p25 and p35 levels in the striatum. (YAC128: $n=8$; WT: $n=8$ ). (B) On the other hand, p25 level is increased in the striatum of YAC128 mice $(n=6)$ compared to WT mice $(n=6)$ following intrastriatal QA injection (unpaired two-tailed $t$-test, $* p=0.0238)$. p35 level was not statistically different between YAC128 $(n=6)$ and WT $(n=6)$ striatum. Therefore, increased susceptibility to QA toxicity in YAC128 mice is associated with increased p25 levels. 
an age where the mice show increased susceptibility to QA neurotoxicity, p25 levelin the striatum is also increased in response to QA.

\section{DISCUSSION}

Previous studies have demonstrated increased NMDA response and calpain activation in 2-month old YAC128 mice MSNs [7, 27]. The neurotoxic cdk5 activator p25 is generated by calpain-mediated cleavage of p35 under excitotoxic conditions [16]. It has been demonstrated that $\mathrm{p} 25$-mediated Cdk5 dysregulation may be involved in neuronal loss in a number of neurodegenerative disorders $[14,15]$ and is thought to result from altered subcellular localization and activity of cdk5/p25 complex [16]. Our results show that a reduction in the $\mathrm{p} 25$ response as a consequence of decreased p35 levels in p35+/- mice reduces NMDAmediated apoptosis of MSNs in culture and mitigates QA-mediated striatal degeneration in vivo. Additionally, we show that QA-induced striatal excitotoxicity results in an elevated p25 level in YAC128 mice compared to WT mice.

Elevated endogenous levels of QA are thought to play a role in HD neurodegeneration [28]. Intrastriatal QA injection recapitulates many neurodegenerative features of HD [25] and the QA level is elevated in the striatum of full-length mHtt HD mouse models [29]. In YAC128 mice, an increase in striatal QA levels is observed around 8-months of age and persists thereafter [29]. This increase precedes neuronal loss observed in these mice [26], suggesting that QA may be involved in mediating neurodegeneration.

Examination of 4-month old YAC128 mice and WT mice in our study show that in the presence of $\mathrm{mHtt}$, the p25 level is elevated in response to intrastriatal QA injection, despite the fact that we did not detect any difference in the basal p25 level between untreated YAC128 and WT mice. It is worth noting that the neuropathology is not pronounced in these mice at this age [26]. However, YAC128 mice do show an increased sensitivity to QA-mediated neurotoxicity at this age and display larger QA-mediated striatal lesions compared to WT mice [27]. Therefore, the elevated p25 level in the striatum in response to QA injection correlates well with the increased sensitivity to QAinduced neurodegeneration in YAC128 mice and may be involved in the striatal neurodegenerative process.

Despite the elevated p25 levels observed in the striatum of YAC128 mice, the ratio of the $150 \mathrm{kDa}$ spectrin cleavage product to the $250 \mathrm{kDa}$ full-length spectrin was not different between QA injected WT and YAC128 mice, suggesting similar levels of calpain activation between the two genotypic groups (suppl. Figure 3). Since p25 is generated from calpainmediated cleavage of p35, the higher p25 level observed in YAC128 despite seemingly similar calpain activity is intriguing. The mechanism by which p 25 is elevated may be one of the underlying mechanisms for neurodegeneration and needs further examination.

It is worth noting that a number of studies have suggested that Htt phosphorylation by cdk5 is neuroprotective in HD [30, 31]. A number of studies also suggest that cdk5 activation by $\mathrm{p} 35$ promotes neuronal survival [13]. As mentioned previously, p35+/mice have reduced levels of both p35 and p25. Therefore, decreased p35 levels in these mice could have resulted in increased sensitivity to excitotoxicity. On the contrary, p35+/- mice showed reduced striatal sensitivity to QA- and NMDA-mediated neurotoxicity, demonstrating that the reduction in p25 and p35 levels are sufficient to impart neuroprotection against striatal excitotoxicity and suggesting that the cdk5 pathway may be a therapeutic target in HD.

Our findings in YAC128 mice demonstrate that in the presence of $\mathrm{mHtt}$, the p25 level is increased in the striatum in response to NMDA-receptor mediated excitotoxicity. It has been shown previously that $\mathrm{p} 53$ levels are increased in the brains of full-length $\mathrm{mHtt}$ transgenic mice and HD patients [32]. p53 phosphorylation by $\mathrm{p} 25$-mediated cdk5 activation was shown to increase p53 stability and activity [33]. Based on these findings, we hypothesize that reducing p25 levels will lead to decreased p53 activity, and will ameliorate striatal neurodegeneration; further studies are necessary to determine the exact role of p25 in HD neurodegeneration.

In conclusion, our findings demonstrate that a decreased levels of the cdk5 activators, p25 and p35, imparts protection against excitotoxic striatal neurodegeneration in mice. Furthermore, elevated p25 level in the striatum of YAC128 mice following excitotoxic insult suggest a role for the cdk5 pathway in HD striatal neurodegeneration. Therefore, therapeutic strategies that modulate the cdk5 pathway may be effective in HD.

\section{ACKNOWLEDGMENTS}

We thank A. Hill, and C. Lefroy for technical assistance and mouse colony maintenance. This work was supported by Pacific Alzheimer Research Foundation (BRL and KHJP) and the CIHR (MOP-12699 to LAR). 


\section{SUPPLEMENTARY MATERIAL}

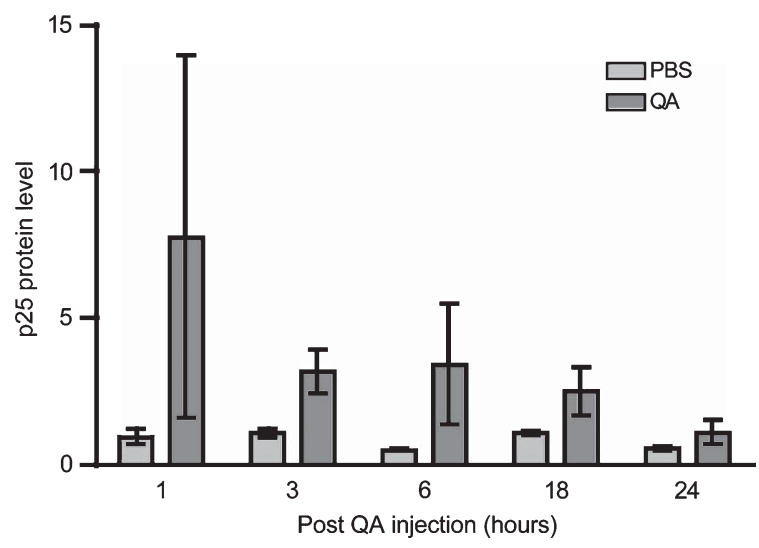

Supplementary Figure 1. Time course analysis of striatal p25 level in FVB WT mice in response to intrastriatal QA injections. Unilaterally injected striatum was examined at various time-points following intrastriatal QA injection. p25 level in the striatum was elevated in response to QA (Two-way ANOVA, treatment effect, $p=0.0498$ ). QA injection samples showed large variability compared to PBS injection samples demonstrating the technical difficulty of intrastriatal QA injection. $n=3$ mice per treatment and time point.

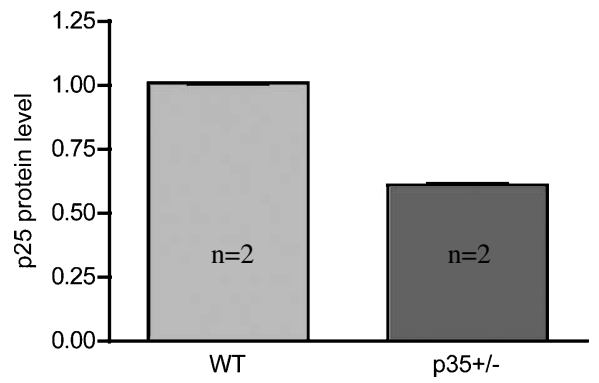

Supplementary Figure 2. p35+/- show reduced p25 levels in striatum in response to QA injection. p35+/- mice show approximately $40 \%$ reduction in $\mathrm{p} 25$ level compared to WT mice. $n=2$ per genotype.

\section{REFERENCES}

[1] Huntington's Disease Collaborative Research Group. A novel gene containing a trinucleotide repeat that is expanded and unstable on Huntington's disease chromosomes. Cell. 1993;72:971-83.

[2] Ferrante R, Kowall N, Bea 1M, Richardson EJ, Bird E, Martin J. Selective sparing of a class of striatal neurons in Huntington's disease. Science. 1985;230(4725):561-3.

[3] Zeron MM, Hansson O, Chen N, Wellington CL, Leavitt $\mathrm{BR}$, Brundin $\mathrm{P}$, et al. Increased sensitivity to N-methyl-Daspartate receptor-mediated excitotoxicity in a mouse model of Huntington's disease. Neuron. 2002;33:849-60.

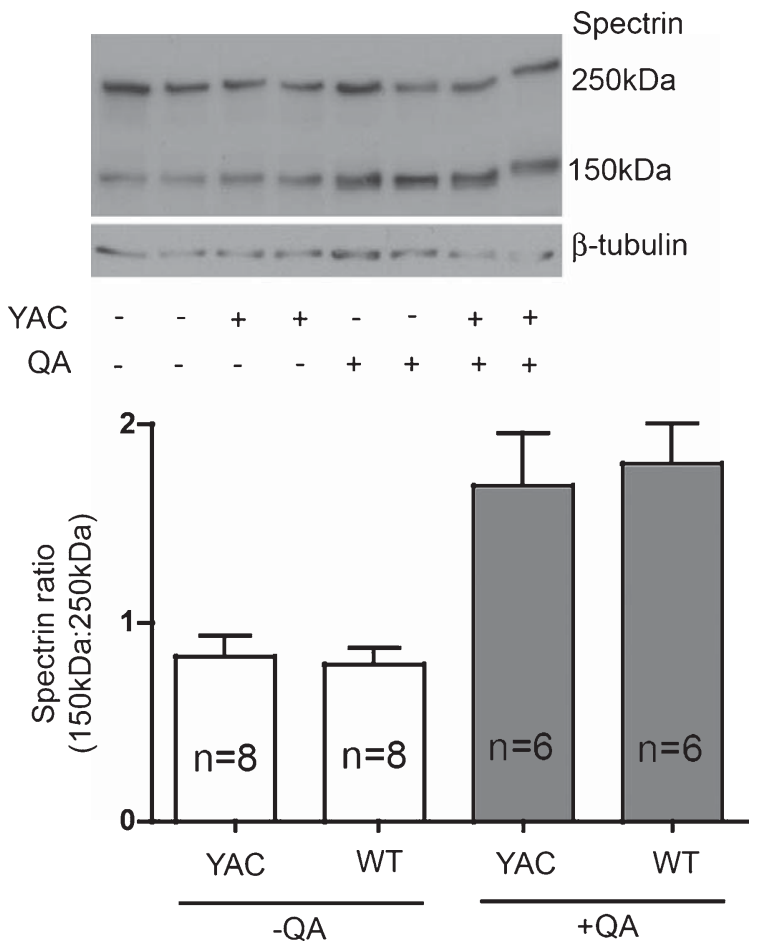

Supplementary Figure 3. Intrastriatal QA injection in YAC128 and WT mice increase spectrin cleavage. Spectrin is a calpain substrate and is cleaved upon calpain activation and generates $150 \mathrm{kDa}$ fragment. Increase in the ratio of $150 \mathrm{kDa}$ to $250 \mathrm{kDa}$ band demonstrates calpain activation in striatum following QA injection. The ratios were not different between YAC128 and WT mice in either untreated (-QA) or treated (+QA) conditions, suggesting that there is no genotypic effect on calpain activation in 4-month old mice examined in our study. (One-way ANOVA, $p<0.0001$; Tukey's multiple comparison test).

[4] Fernandes H, Baimbridge K, Church J, Hayden M, Raymond L. Mitochondrial sensitivity and altered calcium handling underlie enhanced NMDA-induced apoptosis in YAC128 model of Huntington's disease. J Neurosci. 2007;27(50): 13614-23.

[5] Vosler PS, Brennan CS, Chen J. Calpain-mediated signaling mechanisms in neuronal injury and neurodegeneration. Mol Neurobiol. 2008;38:78-100.

[6] Gafni J, Ellerby LM. Calpain activation in Huntington's disease. J Neurosci. 2002;22(12):4842-9.

[7] Cowan CM, Fan MMY, Fan J, Shehadeh J, Zhan LYJ, Graham RK, et al. Polyglutamine-modulated striatal calpain activity in YAC transgenic huntington disease mouse model: impact on NMDA receptor function and toxicity. J Neurosci. 2008;28(48):12725-35. 
[8] Lee KY, Rosales JL, Tang D, Wang JH. Interaction of cyclindependent kinase $5(\mathrm{Cdk} 5)$ and neuronal Cdk5 activator in bovine brain. J Biol Chem. 1996;271(3):1538-43.

[9] Tang D, Yeung J, Lee KY, Matsushita M, Matsui H, Tomizawa $\mathrm{K}$, et al. An isoform of the neuronal cyclin-dependent kinase 5 (Cdk5) activator. J Biol Chem. 1995;270(45):26897-903.

[10] Tsai LH, Delalle I, Caviness VS Jr, Chae T, Harlow E. p35 is a neural-specific regulatory subunit of cyclin-dependent kinase 5. Nature. 1994;371(6496):419-23.

[11] Mao D, Hinds P. p35 is required for CDK5 activation in cellular senescence. J Biol Chem. 2010;285(19):14671-80.

[12] Dhavan R, Tsai LH. A decade of CDK5. Nat Rev Mol Cell Biol. 2001;2(10):749-59.

[13] Hisanaga S, Endo R. Regulation and role of cyclin-dependent kinase activity in neuronal survival and death. J Neurochem. 2010;115:1309-21.

[14] Lopes J, Agostinho P. Cdk5: multitasking between physiological and pathological conditions. Prog Neurobiol. 2011;94(1):49-63.

[15] Cheung Z, Ip N. Cdk5: A multifaceted kinase in neurodegenerative diseases. Trends Cell Biol. 2011.

[16] Patrick GN, Zukerberg L, Nikolic M, de la Monte S, Dikkes P, Tsai LH. Conversion of p35 to p25 deregulates Cdk5 activity and promotes neurodegeneration. Nature. 1999;402(6762):615-22.

[17] Lee MS, Kwon YT, Li M, Peng J, Friedlander RM, Tsai LH. Neurotoxicity induces cleavage of p35 to p25 by calpain. Nature. 2000;405(6784):360-4

[18] Kusakawa G, Saito T, Onuki R, Ishiguro K, Kishimoto T, Hisanaga S. Calpain-dependent proteolytic cleavage of the p35 cyclin-dependent kinase 5 activator to p25. J Biol Chem. 2000;275(22):17166-72

[19] Asada A, Yamamoto N, Gohda M, Saito T, Hayashi N, Hisanaga S. Myristoylation of p39 and p35 is a determinant of cytoplasmic or nuclear localization of active cyclin-dependent kinase 5 complexes. J Neurochem. 2008;106(3):1325-36.

[20] Paoletti P, Vila I, Rifé M, Lizcano JM, Alberch J, Ginés S. Dopaminergic and glutamatergic signaling crosstalk in Huntington's disease neurodegeneration: the role of p25/cyclindependent kinase 5. J Neurosci. 2008;28(40):10090-101.

[21] Crespo-Biel N, Camins A, Pelegrí C, Vilaplana J, Pallàs M, Canudas AM. 3-Nitropropionic acid activates calpain/cdk5 pathway in rat striatum. Neurosci Lett. 2007;421:77-81.

[22] Hallows JL, Chen K, DePinho RA, Vincent I. Decreased cyclin-dependent kinase 5 (cdk5) activity is accompanied by redistribution of cdk5 and cytoskeletal proteins and increased cytoskeletal protein phosphorylation in p 35 null mice. J Neurosci. 2003;23(33):10633-44
[23] Fan. J, Cowan C, Zhang L, Hayden M, Raymond L. Interaction of postsynaptic density protein-95 with NMDA receptors influences excitotoxicity in the yeast artificial chromosome mouse model of Huntington's disease. J Neurosci. 2009;29(35):10928-38.

[24] Park KHJ, Hallows JL, Chakrabarty P, Davies P, Vincent I. Conditional neuronal simian virus $40 \mathrm{~T}$ antigen expression induces Alzheimer-like tau and amyloid pathology in mice. $\mathrm{J}$ Neurosci. 2007;27(11):2969-78.

[25] Beal MF, Kowall NW, Ellison DW, Mazurek MF, Swartz KJ, Martin JB. Replication of the neurochemical characteristics of Huntington's disease by quinolinic acid. Nature. 1986;321:168-71.

[26] Slow EJ, van Raamsdonk J, Rogers D, Coleman SH, Graham RK, Deng Y, et al. Selective striatal neuronal loss in a YAC128 mouse model of Huntington disease. Hum Mol Genet. 2003;12:1555-67.

[27] Graham RK, Pouladi MA, Joshi P, Lu G, Deng Y, Wu N-P, et al. Differential susceptibility to excitotoxic stress in YAC128 mouse models of Huntington disease between initiation and progression of disease. J Neurosci. 2009;29(7):2193204.

[28] Schwarcz R, Guidetti P, Sathyasaikumar KV, Muchowski PJ. Of mice, rats, and men: revisiting the quinolinic acid hypothesis of Huntington's disease. Prog Neurobiol. 2010;90: 230-45.

[29] Guidetti P, Bates GP, Graham RK, Hayden MR, Leavitt BR, MacDonald ME, et al. Elevated brain 3-hydroxykynurenine and quinolinate levels in Huntington disease mice. Neurobiol Dis. 2006;23:190-7.

[30] Luo S, Vacher C, Davies JE, Rubinsztein DC. Cdk5 phosphorylation of huntingtin reduces its cleavage by caspases: Implication for mutant huntingtin toxicity. J Cell Biol. 2005;169(4):647-56.

[31] Anne SL, Saudou F, Humbert S. Phopshorylation of huntingtin by cyclin-dependent kinase 5 is induced by DNA damage and regulates wild-type and mutant huntingtin toxicity in neurons. J Neurosci. 2007;27(27):7318-28.

[32] Bae B-I, Xu H, Igarashi S, Fujimuro M, Agrawal N, Taya Y, et al. p53 mediates cellular dysfunction and behavioral abnormalities in Huntington's disease. Neuron. 2005;47:29-41.

[33] Lee J-H, Kim H-S, Lee S-J, Kim K-T. Stabilization and activation of p53 induced by cdk5 contributes to neuronal cell death. J Cell Sci. 2007;120(13):2259-71. 